\title{
Containment Control for Nonlinear Multi-agent Systems with Parameter Uncertainties and Communication Delays
}

\author{
Ping Wang \\ The Department of Mathematics and Physics, North China Electric Power University, 619 Yonghua North Street \\ Baoding, 100073, China \\ Yingmin Jia \\ The Seventh Research Division and the Center for Information and Control, School of Automation Science and \\ Electrical Engineering, Beihang University (BUAA), 37 Xueyuan Road, Haidian District \\ Beijing, 100191, China \\ E-mail: wangpinglh2014@126.com; ymjia@buaa.edu.cn \\ www.ncepu.edu.cn
}

\begin{abstract}
This paper considers robust containment control problem for uncertain multi-agent systems with inherent nonlinear dynamics. A distributed protocol is proposed using local delayed state information, and then the original problem is converted into an $H_{\infty}$ control problem by defining an appropriate controlled output function. Based on robust $H_{\infty}$ theory, sufficient conditions in terms of linear matrix inequalities (LMIs) are derived to ensure the $H_{\infty}$ containment. A numerical example is provided to demonstrate the effectiveness of our theoretical results.

Keywords: multi-agent systems, containment control, robust $H_{\infty}$ control, inherent nonlinear dynamics.
\end{abstract}

\section{Introduction}

Containment control of multi-agent systems has received considerable attention due to its potential applications, such as earth monitoring, stellar observation for satellite formation ${ }^{1}$, removing hazardous materials for autonomous robots ${ }^{2}$, and so on. Containment control problem arises in the presence of multiple leaders, where its objective is to drive the followers into the convex hull spanned by the leaders.

In engineering practice, multi-agent systems are often subject to parameter uncertainties and external disturbances, which might degrade the system performance and even cause the network system to diverge or oscillate. For such cases, $H_{\infty}$ containment control problems for second-order and uncertain multiagent systems with inherent nonlinear dynamics were considered in Refs. 3 and 4, respectively. Whereas, the effects of communication delays on $H_{\infty}$ containment control are not considered in the existing works.

Motivated by the above analysis, we employ $H_{\circ}$ control approach to investigate the containment control problem of nonlinear multi-agent systems with parameter uncertainties and communication delays. Based on the local delayed state information, a distributed protocol is proposed, and then the original problem is reformulated as an $H_{\infty}$ control problem by defining an appropriate controlled output function. Sufficient conditions in terms of LMIs are derived to ensure the containment performance with a desired $H_{\infty}$ disturbance attenuation level. Moreover, the proposed protocol can be determined by solving only two LMIs with the same dimensions as a single agent. 


\section{Preliminaries and Problem Formulation}

\subsection{Preliminaries}

Directed graphs ${ }^{5}$ are used to model the interaction topologies among agents. Suppose that a multi-agent system consists of $M$ followers and $N-M$ leaders. An agent is called a leader if the agent has no neighbor, and a follower if the agent has at least one neighbor. Due to the fact that the leaders have no neighbor, the Laplacian matrix $L$ associated with graph $G$ can be partitioned as

$$
L=\left[\begin{array}{cc}
L_{1} & L_{2} \\
0_{(N-M) \times M} & 0_{(N-M) \times(N-M)}
\end{array}\right]
$$

where $L_{1} \in R^{M \times M}$ and $L_{2} \in R^{M \times(N-M)}$.

Assumption 1. Suppose that for each follower, there exists at least one leader that has a directed path to that follower. Moreover, the interaction topology among the $M$ followers is undirected.

Lemma 1. ${ }^{6}$ Under Assumption 1, $L_{1}$ is positive definite, $-L_{1}^{-1} L_{2}$ is nonnegative, and the sum of the entries in each row equals 1 .

\subsection{Problem formulation}

Consider the multi-agent system consisting of $M$ followers and $N-M$ leaders. We use $F \square\{1, \cdots, M\}$ and $R \square\{M+1, \cdots, N\}$ to denote the follower set and the leader set, respectively. Each follower with nonlinear dynamics is represented as

$\dot{x}_{i}(t)=A x_{i}(t)+B_{1} u_{i}(t)+B_{2} \omega_{i}(t)+B_{3} f\left(t, x_{i}(t)\right), \quad i \in F$

where $x_{i}(t) \in R^{m}, u_{i}(t) \in R^{m_{1}}$ are the state and the control input (or protocol), respectively, $\omega_{i}(t) \in R^{m_{2}}$ is the external disturbance that belongs to $L_{2}[0, \infty)$, and $f: R \times R^{m} \rightarrow R^{m}$ is the inherent nonlinear dynamics of the $i$ th follower.

The leaders of the concerned multi-agent system are described by

$$
\dot{x}_{i}(t)=A x_{i}(t)+B_{3} f\left(t, x_{i}(t)\right), i \in R
$$

where $x_{i}(t) \in R^{m}$ is the state of the leader $i$.

Assumption 2. Given $\eta_{1}, \cdots, \eta_{N-M}$ with $\sum_{i=1}^{N-M} \eta_{i}=1$ and $\eta_{i} \geq 0, i=1, \cdots, N-M$. There exists a nonnegative constant $\rho$ such that nonlinear function $f(t, x(t))$ satisfies

$\left\|f(t, x(t))-\sum_{i=1}^{N-M} \eta_{i} f\left(t, y_{i}(t)\right)\right\| \leq \rho\left\|x(t)-\sum_{i=1}^{N-M} \eta_{i} y_{i}(t)\right\|$ $\forall x, y_{i} \in R^{m}, i=1,2, \cdots N-M, \forall t \geq 0$.

When system matrices $A, B_{1}, B_{2}, B_{3}$ are uncertain, they are assumed to be of the following form

$$
\begin{array}{cl}
A=A_{0}+\Delta A(t), & B_{1}=B_{10}+\Delta B_{1}(t), \\
B_{2}=B_{20}+\Delta B_{2}(t), & B_{3}=B_{30}+\Delta B_{3}(t)
\end{array}
$$

where $A_{0}, B_{10}, B_{20}, B_{30}$ are known real constant matrices, $\Delta A(t), \Delta B_{1}(t), \Delta B_{2}(t), \Delta B_{3}(t)$ are unknown real normbounded matrix functions satisfying

$\left[\Delta A(t), \Delta B_{1}(t), \Delta B_{2}(t), \Delta B_{3}(t)\right]=E \Sigma(t)\left[F_{1}, F_{2}, F_{3}, F_{4}\right](6)$ with $E, F_{1}, F_{2}, F_{3}$ and $F_{4}$ being known constant matrices, and $\Sigma(t)$ being an unknown real time-varying matrix that satisfies $\Sigma^{T}(t) \Sigma(t) \leq I$. It is also assumed that $\left(A_{0}, B_{10}\right)$ is stabilized.

Define a controlled output function $z(t)=\left[z_{1}^{T}(t), z_{2}^{T}(t), \cdots, z_{M}^{T}(t)\right]^{T}$ with

$$
z_{i}(t)=x_{i}(t)-y_{i}(t), \quad i \in F, y_{i}(t) \in \Omega(t)
$$

to quantitatively analyze the effect of external disturbances on the containment control problem, where, $\Omega(t)=\left\{\sum_{i=M+1}^{N} \alpha_{i} x_{i} \mid \alpha_{i} \in R, \alpha_{i} \geq 0, \sum_{i=M+1}^{N} \alpha_{i}=1\right\}$. Obviously, if $\lim z(t)=0$, the containment control problem of the multi-agent system (2)-(3) can be solved. Thus, the attenuating ability of multi-agent system against external disturbances can be quantitatively measured by

$$
\left\|T_{z \omega}(s)\right\|_{\infty}=\sup _{0 \neq \omega(t) \in L_{2}[0, \infty)} \frac{\|z(t)\|_{2}}{\|\omega(t)\|_{2}}
$$

where $\omega(t)=\left[\omega_{1}^{T}(t), \cdots, \omega_{M}^{T}(t)\right]^{T},\|z(t)\|_{2}^{2}=\int_{0}^{\infty} z^{T}(t) z(t) d t$, $\|\omega(t)\|_{2}^{2}=\int_{0}^{\infty} \omega^{T}(t) \omega(t) d t, T_{z \omega}(s)$ represents the closedloop transfer function matrix from the external disturbance $\omega(t)$ to the controlled output $z(t)$.

\section{Main Results}

\subsection{Protocol design and problem reformulation}

A distributed protocol is proposed using local delayed state information.

$$
u_{i}(t)=K \sum_{j \in F \cup R} a_{i j}\left[x_{j}(t-\tau(t))-x_{i}(t-\tau(t))\right], i \in F
$$

where $K$ is a feedback gain matrix to be determined later, $\tau(t)$ is the time-varying communication delay which satisfies $0 \leq \tau(t) \leq h, \forall t \geq 0$.

Denote

$$
x_{f}(t)=\left[x_{1}^{T}(t), \cdots, x_{M}^{T}(t)\right]^{T}, x_{l}(t)=\left[x_{M+1}^{T}(t), \cdots, x_{N}^{T}(t)\right]^{T},
$$




$$
\begin{aligned}
& F\left(t, x_{f}(t)\right)=\left[f^{T}\left(t, x_{1}(t)\right), \cdots, f^{T}\left(t, x_{M}(t)\right)\right]^{T}, \\
& F\left(t, x_{l}(t)\right)=\left[f^{T}\left(t, x_{M+1}(t)\right), \cdots, f^{T}\left(t, x_{N}(t)\right)\right]^{T} .
\end{aligned}
$$

Let $\bar{x}(t)=x_{f}(t)-\left(-L_{1}^{-1} L_{2} \otimes I_{m}\right) x_{l}(t), \quad$ the closed-loop system (2) and (3) under protocol (9) can be written as

$$
\begin{aligned}
\dot{\bar{x}}(t)= & \left(I_{M} \otimes A\right) \bar{x}(t)-\left(L_{1} \otimes B_{1} K\right) \bar{x}(t-\tau(t)) \\
& +\left(I_{M} \otimes B_{2}\right) \omega(t)+\left(I_{M} \otimes B_{3}\right) \bar{F}(t)
\end{aligned}
$$

where $\bar{F}(t)=F\left(t, x_{f}(t)\right)+\left(L_{1}^{-1} L_{2} \otimes I_{m}\right) F\left(t, x_{l}(t)\right)$.

By Lemma $1,-\left(L_{1}^{-1} L_{2} \otimes I_{m}\right) x_{l}(t)$ is in the convex hull spanned by the leaders. In this paper, the controlled output function $z(t)$ is defined as

$$
z(t)=\bar{x}(t) .
$$

Until now, the containment control problem can be reformulated as the following $H_{\infty}$ control problem

$$
\begin{aligned}
\dot{\bar{x}}(t)= & \left(I_{M} \otimes A\right) \bar{x}(t)-\left(L_{1} \otimes B_{1} K\right) \bar{x}(t-\tau(t)) \\
& +\left(I_{M} \otimes B_{2}\right) \omega(t)+\left(I_{M} \otimes B_{3}\right) \bar{F}(t) \\
z(t)= & \bar{x}(t) .
\end{aligned}
$$

Therefore, if the system (11) is asymptotically stable with $\left\|T_{z \omega}(s)\right\|_{\infty}<\gamma \quad$ satisfied, then $H_{\infty}$ containment control for the multi-agent system (2)-(3) can be solved.

\subsection{Conditions for $H_{\circ}$ containment performance}

Lemma 2. Suppose that Assumptions 1 and 2 hold. System (11) is asymptotically stable with $\left\|T_{z \omega}(s)\right\|_{\infty}<\gamma$ satisfied, if there exist positive definite matrices $P, R \in R^{m \times m}$ such that the inequality

$\left[\begin{array}{cccccc}\Psi_{11}^{i} & \Psi_{12}^{i} & P B_{2} & P B_{3} & I_{m} & \Psi_{16}^{i} \\ * & -R & 0_{m \times m_{2}} & 0_{m} & 0_{m} & \Psi_{26}^{i} \\ * & * & -\gamma^{2} I_{m_{2}} & 0_{m_{2} \times m} & 0_{m_{2} \times m} & h B_{2}^{T} R \\ * & * & * & -I_{m} & 0_{m} & h B_{3}^{T} R \\ * & * & * & * & -\frac{I_{m}}{\rho^{2}+1} & 0_{m} \\ * & * & * & * & * & -R\end{array}\right]<0$

$\Psi_{11}^{i}=P A+A^{T} P-\lambda_{i}\left(P B_{1} K+K^{T} B_{1}^{T} P\right)$,

$\Psi_{12}^{i}=\lambda_{i} P B_{1} K, \Psi_{16}^{i}=h\left(A^{T}-\lambda_{i} K^{T} B_{1}^{T}\right) R$,

$\Psi_{26}^{i}=h \lambda_{i} K^{T} B_{1}^{T} R$

is simultaneously satisfied for all $i \in F$, where $\lambda_{i}$ is the $i$ th eigenvalue of the matrix $L_{1}$.

Theorem 1. Suppose that Assumptions 1 and 2 hold. Protocol (9) solves the containment control problem with $\left\|T_{z \omega}(s)\right\|_{\infty}<\gamma$ satisfied, if there exist a positive definite matrix $X \in R^{m \times m}$, a matrix $W \in R^{m_{1} \times m}$ and positive scalars $c$ and $\eta$ such that the following LMI

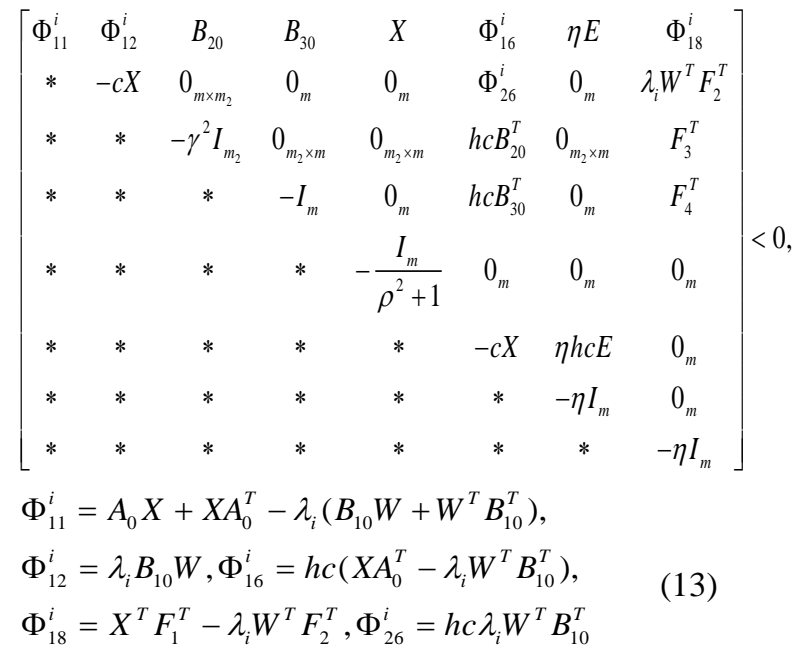

$i$ simultaneously satisfied for $i=1$ and $M$. Moreover, when the above two LMIs are feasible, the feedback matrix of the protocol (9) is designed as $K=W X^{-1}$.

Proof. Denote

$\bar{F}=\left[E^{T} P^{T}, 0_{m}, 0_{m \times m_{2}}, 0_{m}, 0_{m}, h c E^{T} P^{T}\right]^{T}$,

$\bar{H}=\left[F_{1}-\lambda_{i} F_{2} K, \lambda_{i} F_{2} K, F_{3}, F_{4}, 0_{m}, 0_{m}\right], i \in F$.

$$
\begin{aligned}
\tilde{\Psi}^{i}= & {\left[\begin{array}{cccccc}
\tilde{\Psi}_{11}^{i} & \tilde{\Psi}_{12}^{i} & P B_{20} & P B_{30} & I_{m} & \tilde{\Psi}_{16}^{i} \\
* & -R & 0_{m \times m_{2}} & 0_{m} & 0_{m} & \tilde{\Psi}_{26}^{i} \\
* & * & -\gamma^{2} I_{m_{2}} & 0_{m_{2} \times m} & 0_{m_{2} \times m} & h B_{20}^{T} R \\
* & * & * & -I_{m} & 0_{m} & h B_{30}^{T} R \\
* & * & * & * & -\frac{I_{m}}{\rho^{2}+1} & 0_{m} \\
* & * & * & * & * & -R
\end{array}\right] } \\
\tilde{\Psi}_{11}^{i}= & P A_{0}+A_{0}^{T} P-\lambda_{i}\left(P B_{10} K+K^{T} B_{10}^{T} P\right), \\
\tilde{\Psi}_{12}^{i}= & \lambda_{i} P B_{10} K, \tilde{\Psi}_{16}^{i}=h\left(A_{0}^{T}-\lambda_{i} K^{T} B_{10}^{T}\right) R, \\
\tilde{\Psi}_{26}^{i}= & h \lambda_{i} K^{T} B_{10}^{T} R .
\end{aligned}
$$

Substituting (5) and (6) into (12) results in the following inequality

$$
\tilde{\Psi}^{i}+\bar{F} \Sigma(t) \bar{H}_{i}+\bar{H}_{i}^{T} \Sigma^{T}(t) \bar{F}^{T}<0 .
$$

Due to the fact that $\Sigma^{T}(t) \Sigma(t) \leq I$, inequality (14) holds if and only if there exists a scalar $\eta>0$ such that

$$
\tilde{\Psi}^{i}+\eta \bar{F} \bar{F}^{T}+\eta^{-1} \bar{H}_{i}^{T} \bar{H}_{i}<0 .
$$

By Schur Complement, inequality (15) can be transformed to a matrix inequality. Let $R=c P, c>0$. Pre- and post-multiplying the above inequality with 
$\Gamma=\operatorname{diag}\left\{P^{-1}, P^{-1}, I_{m_{2}}, I_{m}, I_{m}, P^{-1}, I_{m}, I_{m}\right\}$ and $\Gamma^{T}$, respectively, and then denoting $X=P^{-1}, K P^{-1}=W$, we can obtain an equivalent inequality (13), which is an LMI with respect to variable $W$ and $X$. Due to the fact that $0<\lambda_{1} \leq \lambda_{2} \leq \cdots \leq \lambda_{M}$, in view of its convex property, when LMI (13) holds for $\lambda_{1}$ and $\lambda_{M}$ simultaneously, it holds for all $\lambda_{i}, i \in F$. By Lemma 2, the containment control problem can be solved with $\left\|T_{z \omega}(s)\right\|_{\infty}<\gamma$ satisfied.

\section{Simulation}

Consider a network consisting of four followers and two leaders, whose dynamics are described by (2) and (3) respectively, with

$$
\begin{aligned}
& A_{0}=\left[\begin{array}{cc}
0 & -1 \\
2 & 1
\end{array}\right], B_{10}=\left[\begin{array}{ll}
1 & 0 \\
0 & 2
\end{array}\right], B_{20}=\left[\begin{array}{l}
0 \\
1
\end{array}\right], B_{30}=\left[\begin{array}{ll}
0 & 0 \\
0 & 1
\end{array}\right], \\
& E=\left[\begin{array}{cc}
0 & 0 \\
0.2 & 0.2
\end{array}\right], \Sigma(t)=\left[\begin{array}{cc}
\sin (t) & 0 \\
0 & 1-e^{-t}
\end{array}\right], F_{1}=\left[\begin{array}{cc}
0.1 & 0 \\
0 & 0.1
\end{array}\right], \\
& F_{2}=\left[\begin{array}{cc}
0.2 & 0 \\
0 & 0.2
\end{array}\right], F_{3}=\left[\begin{array}{l}
0.3 \\
0.3
\end{array}\right], F_{2}=\left[\begin{array}{cc}
0.4 & 0 \\
0 & 0.4
\end{array}\right], \\
& x_{i}(t)=\left[\begin{array}{c}
x_{i 1}(t) \\
x_{i 2}(t)
\end{array}\right], f\left(t, x_{i}(t)\right)=\left[\begin{array}{c}
0.5 \sin (t) x_{i 1}(t) \\
0.5 \cos (t) x_{i 2}(t)
\end{array}\right] .
\end{aligned}
$$

The external disturbance is supposed to be $\omega(t)=[\psi(t), 1.5 \psi(t), 2 \psi(t),-\psi(t)]^{T}$, where $\psi(t)$ is the band-limited white noise with noise power 0.5 , whose action time interval is $[0,2]$.

Fig.1. shows the communication graph with 0-1 weights, where nodes 5 and 6 are two leaders and the others are followers. The smallest and largest eigenvalues of the matrix $L_{1}$ are $\lambda_{1}=0.3820$ and $\lambda_{4}=3.6180$, respectively.

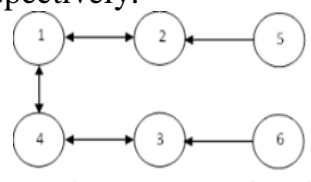

Fig.1. The communication graph

Take $\rho=0.5, c=30$, and $\gamma=1$. Solving LMI (13) related to $\lambda_{1}$ and $\lambda_{4}$, we obtain the maximal allowable delay $h \leq 0.042$. Here we take the communication delay in networks as $\tau(t)=0.04 \sin ^{2}(t)$.

When there are no external disturbances, the initial states of the followers are chosen randomly from the box $[10,30] \times[-5,5]$, and the leaders' initial states are given as $x_{5}(0)=[15,-1]^{T}$ and $x_{6}(0)=[20,1]^{T}$, respectively. Fig.2. give the state trajectories of the network and the energy relationship between $z(t)$ and $\omega(t)$, respectively. Clearly, the containment control problem is indeed solved with $\left\|T_{z \omega}\right\|<1$ satisfied.
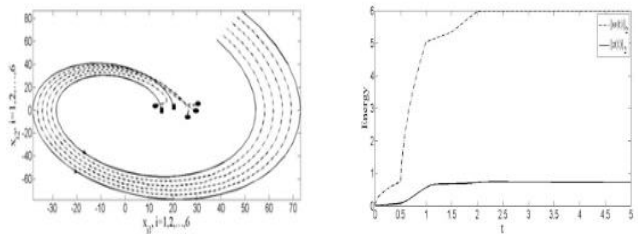

Fig.2. Left: trajectories of the network. Right: trajectories of $z(t)$ and $\omega(t)$

\section{Conclusions}

In this paper, we have employed $H_{\infty}$ control approach to solve the containment control problem for nonlinear multi-agent systems with parameter uncertainties and communication delays. Sufficient conditions in terms of LMIs have been derived to ensure the $H_{\infty}$ containment performance. Moreover, the proposed protocol has been designed by solving only two LMIs with the same dimensions as a single agent.

\section{Acknowledgements}

This work was supported by the National Basic Research Program of China (973 Program: 2012CB821200, 2012CB821201), the NSFC (61134005, 61521091, 61327807) and the Fundamental Research Funds for the Central Universities 2016MS129.

\section{References}

1. D.V. Dimarogonas, P. Tsiotras and K.J. Kyriakopoulos, Leader-follower cooperative attitude control of multiple rigid bodies, Syst. Control Lett., 58(6) (2009) 429-435.

2. G. Notarstefano, M. Egerstedt and M. Haque, Containment in leader-follower networks with switching communication topologies, Automatica, 47(5) (2011) $1035-1040$

3. P. Wang and Y. Jia, Robust $H_{\infty}$ containment control for second-order multi-agent systems with nonlinear dynamics in directed networks, Neurocomputing, 153 (2015) 235-241.

4. P. Wang and Y. Jia, Robust $H_{\infty}$ containment control for uncertain multi-agent systems with inherent nonlinear dynamics, Int. J. Syst. Sci., 47(5) (2016) 1073-1083.

5. C. Godsil and G. Royle, Algebraic Graph Theory, (Springer-Verlag, New York, 2001).

6. W. Ren and Y. Cao, Distributed Coordination of Multiagent Networks: Emergent Problems, Models and Issues, (Springer-Verlag, London, 2011). 